\title{
Expeditious building of ring-porous earlywood vessel chronologies without loosing signal information
}

\author{
Patrick Fonti - Britta Eilmann • \\ Ignacio García-González · Georg von Arx
}

Received: 27 April 2008/Revised: 28 November 2008/Accepted: 15 December 2008/Published online: 6 January 2009

(C) Springer-Verlag 2009

\begin{abstract}
Chronologies of earlywood vessel size of ringporous trees contain valuable ecological information, but long preparation procedures limit their application in ecological studies. Recent and fast techniques for wood surface preparation combined with automated image analysis are reducing the work needed to build chronologies, but might also entail measurement inaccuracy. In this study, we aim to evaluate the effect of a possible efficiency-accuracy trade-off on ecological signal strength. To this end, we compare measurements of mean vessel area
\end{abstract}

Communicated by M. Buckeridge.

P. Fonti $(\bowtie)$

Dendro Sciences Unit, Swiss Federal Research Institute WSL, Zürcherstrasse 111, 8903 Birmensdorf, Switzerland

e-mail: patrick.fonti@wsl.ch

B. Eilmann

Forest Dynamics Unit, Swiss Federal Research Institute WSL, Zürcherstrasse 111, 8903 Birmensdorf, Switzerland

e-mail: britta.eilmann@wsl.ch

I. García-González

Departamento de Botánica,

Universidade de Santiago de Compostela, Escola Politécnica

Superior, Campus de Lugo, 27002 Lugo, Spain

e-mail: ignacio.garcia@usc.es

G. von Arx

Laboratory of Tree Ring Research,

University of Arizona,

105 West Stadium, Tucson, AZ 85721-0058, USA

e-mail: vonarx@1trr.arizona.edu

G. von Arx

School of Natural Resources, University of Arizona,

Biological Sciences East, Tucson, AZ 85721-0058, USA from two recent and fast procedures carried out on sanded wood surfaces with a reference procedure based on an accurate survey from thin sections. Measurements were performed on increment cores of 15 sessile oaks (Quercus petraea (Mattuschka) Liebl.) for the period 1956-2006. Dissimilarities in results with the reference procedure were quantified and evaluated. Our data show that the workload can be reduced by more than 20 -fold when using the highly automated procedure. Signal weakening caused by measurement errors is negligible for vessels $>6,000 \mu \mathrm{m}^{2}$ and can be easily compensated by increasing the sample size. Manual correction of misrecognized vessels hardly reduced this error further. The new procedures constitute a major step towards an efficient and accurate analysis of earlywood vessel chronologies of ring-porous tree species.

Keywords Dendrochronology ·

Quantitative wood anatomy - Image analysis .

Core surface preparation $\cdot$ ROXAS

\section{Introduction}

Chronologies of wood anatomical features contain original environmental information, thus becoming a valuable tool for ecologists to reconstruct past environmental conditions. For example, St George et al. (2002) reconstructed flooding events based on anomalies in size and number of Quercus macrocarpa Michx. vessels, and Panyushkina et al. (2003) were able to identify a temperature signal from the annual variation of the lumen diameter and wall thickness from tracheids of Larix cajanderi Mayr. For ring-porous tree species, the mean vessel area (MVA) in the earlywood is a useful variable to provide information about past climate which is not equally well retained by the radial increment 
(e.g., Pumijumnong and Park 1999; García-González and Eckstein 2003; Fonti and García-González 2004).

Despite many promising results, the building of wood anatomical chronologies is very time-consuming and hence a major obstacle for further exploration and for a widespread use of this source of environmental information. Improved wood surface preparation and technological advancements in image acquisition and analysis allow partly automated, quicker and cheaper ways for cell measurements (e.g., Jagel and Telewski 1990; Sass and Eckstein 1995; Park and Telewski 1993; Evans et al. 1995; Munro et al. 1996; Spiecker et al. 2000). This increase in efficiency makes the study of wood anatomical features more attractive, but whether and to what extent the accuracy of the survey is hampered with these new techniques still remains unknown.

In this study, we intend to evaluate if there is a loss in the strength of the ecological signal in ring-porous trees due to automated measurement procedures. In particular, by comparing vessel measurements conducted directly on the wood surface of increment cores of sessile oak (Quercus petraea (Mattushka) Liebl.) with reference measurements from thin sections, we aim at (1) quantifying the inaccuracy by identify the minimum size threshold for an adequate recognition of the vessels, (2) assess the influence of misrecognized vessels when performing a highly automated measurement, and (3) evaluate if an additional number of trees can mitigate any inaccuracyinduced weakening in the ecological signal.

\section{Materials and methods}

Sampling and crossdating of tree-rings

The work was carried out on increment cores from 15 dominant, at least 100 years old $Q$. petraea trees growing in a forest near Zurich, Switzerland (latitude $46^{\circ} 23^{\prime} \mathrm{N}$, longitude $8^{\circ} 27^{\prime} \mathrm{E}$ ). From each tree, two $5 \mathrm{~mm}$ diameter cores were collected at breast height and perpendicular to the slope direction at each side of the stem.

The tree-ring widths were first measured to build the corresponding time series (Lintab digital positioning table; Rinntech Heidelberg, Germany), and then visually crossdated to assign each ring to the corresponding calendar year (TSAP; Rinntech Heidelberg, Germany). We finally used the COFECHA software (Holmes 1983) to statistically verify the tree ring dating accuracy.

Preparation of core surface and thin sections

Different procedures of core surface preparation and image acquisition have been considered (SCANNER,

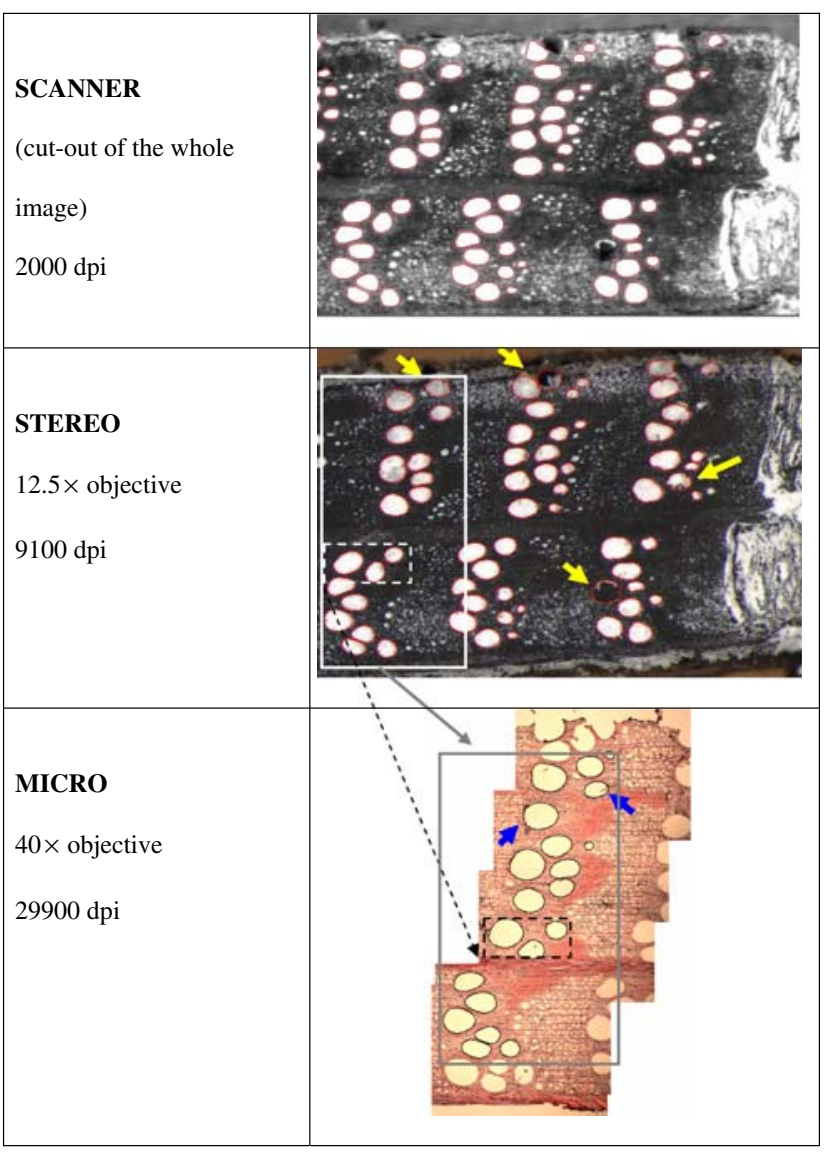

Fig. 1 Comparison of images taken by different procedures. Recognized and measured vessels $>10,000 \mu \mathrm{m}^{2}$ are outlined. The core width is $5 \mathrm{~mm}$. Arrows indicate example where manual corrections have to be applied. The dashed boxes indicate an example of differences in vessel position and size due to the vertical shift along the stem axis

STEREO and MICRO, Fig. 1; Table 1). For the fast measurement procedures (SCANNER and STEREO), each core was sanded using $30 \mu \mathrm{m}$ grit and cleaned with high pressure water blast to remove both tyloses and wood dust from the vessel lumina. In order to improve the contrast, the surrounding tissue was stained black with printer ink (Canon Cartridge BCI-6BK, Canon Inc., Japan) and vessel lumina were filled with white chalk powder.

Thin sections for MICRO were prepared only after image acquisition for the previous procedures had been completed. For this, two cores from one tree were subdivided into pieces of approximately $1.5-2 \mathrm{~cm}$ length. Thin sections (thickness of $10 \mu \mathrm{m}$ ) were then cut from each piece with a sliding microtome (Reichert, Germany), stained with safranin (1\%) and astrablue (2\%), dehydrated with ethanol (70, 95 and 100\%) and xylol, and fixed on microscope slides with Canada balsam (Schweingruber 2001). 
Table 1 Overview of the procedures and their requirements in time and disk space for measuring vessels in one core (51 annual rings)

\begin{tabular}{|c|c|c|c|c|c|}
\hline \multirow[t]{2}{*}{ Procedures } & \multicolumn{4}{|l|}{ Fast } & \multirow{2}{*}{$\begin{array}{l}\text { Reference } \\
\text { MICRO }\end{array}$} \\
\hline & SCANNER & STEREO-auto & & STEREO-manual & \\
\hline \multicolumn{6}{|l|}{ Preparation } \\
\hline Processes & \multicolumn{4}{|c|}{ Sanding, cleaning, dying black and chalk filling } & $\begin{array}{l}\text { Piecing, thin-sectioning, } \\
\text { staining and fixing }\end{array}$ \\
\hline Time & $20 \mathrm{~min}$ & & & & $8 \mathrm{~h}$ \\
\hline \multicolumn{6}{|c|}{ Images acquisition } \\
\hline No. of images & 1 for the whole core & & 1 per ring & & $1-8$ per ring \\
\hline Magnification & $1 \times$ & & $12.5 \times$ & & $40 \times$ \\
\hline Resolution & $2,000 \mathrm{dpi}$ & & 9,100 dpi & & 29,900 dpi \\
\hline Disk space & $6-8 \mathrm{MB}$ & & $350 \mathrm{MB}$ & & $500 \mathrm{MB}$ \\
\hline Time & $10 \min$ & & $50 \min$ & & $6 \mathrm{~h}$ \\
\hline \multicolumn{6}{|l|}{ Measurement } \\
\hline Step unit & Whole core & Ring by ring & & Ring by ring & Ring by ring \\
\hline Mode & Automatic & Automatic & & With manual correction & With manual correction \\
\hline Time & $20 \mathrm{~min}$ & $1 \mathrm{~h}$ & & $2 \mathrm{~h}$ & $6 \mathrm{~h}$ \\
\hline Total time & $50 \mathrm{~min}$ & $2 \mathrm{~h} 10 \mathrm{~min}$ & & $3 \mathrm{~h} 10 \mathrm{~min}$ & $20 \mathrm{~h}$ \\
\hline
\end{tabular}

Time estimates are based on a selection of vessels $>6,000 \mu \mathrm{m}^{2}$

Image acquisition

For SCANNER, images of the entire core were captured using a high-resolution and distortion-free digital scanner (Epson Expression 10000 XL, Seiko Epson Corporation, Japan). The images were scanned on a 256 gray scale level with a resolution of 2,000 dpi, after preliminary tests showed that a higher resolution did not supply any differences in measurement (data not shown).

For STEREO, images (RGB, color 24 bit) were captured ring by ring using a digital video camera (ColorView III, Soft Imaging System, Germany) connected to a stereomicroscope (Leica MZ 12, Leica Microsystems, Germany) with a $12.5 \times$ objective (image resolution: ca. 9,100 dpi).

The same camera was mounted on a transmitted light microscope (Olympus BX41, Japan) to capture images (40× magnification, RGB, 24 bit, ca. 29,900 dpi) of the thin sections (MICRO). Since the field of view through the objective was not large enough to cover the entire tangential width of the core surface, adjacent images from the same annual ring were merged together (Adobe Photoshop Elements 2.0).

\section{Vessel measurement}

Using digital images of cross-sections obtained by each procedure, we measured all vessels from 1956 to 2006, and calculated the MVA for each ring. The measurement of MVA was performed using the image analysis software Image Pro Plus (v4.5, Media Cybernetics, USA). Images captured for SCANNER were analyzed using a self- developed image analysis tool ("ROXAS"; cf. von Arx and Dietz 2005) that combines the functionality of Image Pro Plus with our own code for an automated detection of vessels and tree-ring boundaries. During analysis, ROXAS locally improves and homogenizes image contrast that varies due to natural heterogeneity in wood surface quality. After additional edge enhancement, the images are segmented into a binary image using a fixed threshold value of intensity. Clustered image objects are separated using the "auto split"-function and vessels identified based on area $\left(>1,000 \mu \mathrm{m}^{2}\right)$ and morphometric characteristics. Finally, ring- and vessel-related data are saved into a spreadsheet file. This fully automatic analysis performed the measurement of an entire core in one single step and was thus the fastest of all procedures considered. Misidentified ring boundaries were corrected using a manual editing mode available in ROXAS, but no correction of missing or misidentified vessels was made.

For STEREO and MICRO, vessel recognition was performed ring by ring with the Image Pro Plus-function "automatic [recognition of] bright objects", size filtering $\left(>1,000 \mu \mathrm{m}^{2}\right)$, and an "auto split"-function to separate clustered vessels. Before saving the measurement, recognized vessel perimeters were smoothed ("smoothing" factor $=20$ ) to provide vessels with a more realistic, round outline. Images obtained with STEREO were measured twice: first only automatically (STEREO-auto) and then by correcting misrecognized vessels manually (STEREOmanual). Such corrections consisted of splitting clustered vessels, adjusting (by adding, filling or deleting) misrecognized vessels and excluding incomplete vessels at the edge of 
the cores. The same criteria for manual vessel correction were applied to the reference procedure (MICRO).

Quantifying inaccuracy

The accuracy of vessel survey was evaluated by comparing the MVA values of the fast procedures (SCANNER, STEREO-auto and STEREO-manual) with the reference procedure (MICRO). The (lowest) size threshold above which vessels were recognized with an adequate accuracy was evaluated by monitoring Pearson's correlation coefficient $(r)$ between the reference (MICRO) and the series of the fast procedures (SCANNER, STEREO-auto and STEREO-manual) while gradually increasing the minimum vessel size from 1,000 to $30,000 \mu \mathrm{m}^{2}$. The threshold size was identified when correlations stabilized.

Likewise, the effect of manual corrections on the measurement accuracy (STEREO-auto vs. STEREO-manual) was evaluated by quantifying the improvement of the correlation with the reference.

Estimating the changes in signal information

The loss of information due to measurement inaccuracies was evaluated by comparing the signal properties of the fastest original MVA chronology (SCANNER) with those of the same chronology in which an additional measurement error (ME) was introduced. The measurement error was calculated as the percentage difference in MVA values measured with the SCANNER procedure and the reference (MICRO). After verifying normality (KolmogoroffSmirnoff, $P>0.05$ ), the distribution of percentage difference values was shifted to yield a mean value of 0 . The error-introduced MVA chronology was created by adding to each single original MVA value a randomly generated ME value out of the error distribution. This analysis was performed for SCANNER only, because, due to the largest error, we considered this to be the most conservative approach.

Chronologies of MVA were built by averaging detrended individual time series, after fitting a cubic smoothing spline (32 years stiffness, $50 \%$ frequency cutoff) to each raw series and dividing each value by this function (Cook et al. 1992) to obtain growth indices. After that, we calculated the mean sensitivity (i.e., mean percentage change from each measured yearly ring value to the next) and established climate-growth relationships by correlating the chronologies to monthly meteorological data (Fritts 2001). As signal strength we considered the common signal (i.e., the cross-correlations between all possible pairs of individuals (Rbt) after Briffa and Jones (1992)) and the climatic signal. A previous screening for best climatic variable revealed that MVA significantly correlated to April-May precipitation $(P<0.01, n=51$, meteo data from Zurich, MeteoSwiss, Switzerland).

To evaluate if any loss in the signal could be compensated by a larger sample size, the analyses were performed by progressively increasing the number of trees used for the chronologies. Both common and climatic signals were computed by bootstrapping $(n=1,000)$ the 15 trees with replacement, for a number of two up to all 15 trees. For each repetition, we used a newly-created set of error-introduced chronologies. Only MVA time series with vessels larger than $10,000 \mu \mathrm{m}^{2}$ were considered for these comparisons, a threshold previously recommended for dendroecological analyses of MVA (García-González and Fonti 2006).

\section{Results}

General differences among procedures

The time needed for measuring the vessels differed strongly among procedures (Table 1). If only vessels $>6,000 \mu \mathrm{m}^{2}$ were taken into account, SCANNER accelerated the vessel survey by about 24 times in comparison with MICRO, the most demanding measurement procedure in all processes (preparation, image acquisition and measurement). But the procedures also differed in their output when all vessel sizes were considered, in particular for the number of vessels recognized (Table 2). In MICRO, five to seven times more vessels per ring were recognized than in
Table 2 Size distribution (mean \pm 1 standard deviation) of the number of recognized vessels per annual ring

Values correspond to measurements performed on two cores of the same tree $(n=102)$

\begin{tabular}{lcccc}
\hline & \multicolumn{3}{l}{ Procedure } & \\
\cline { 2 - 5 } Size class $\left(\mu \mathrm{m}^{2}\right)$ & SCANNER & STEREO-auto & STEREO-manual & MICRO \\
\hline All vessels $(>1,000)$ & $103 \pm 24$ & $141 \pm 33$ & $134 \pm 33$ & $748 \pm 164$ \\
$1,000-5,000$ & $43 \pm 16$ & $82 \pm 26$ & $75 \pm 26$ & $680 \pm 162$ \\
$5,000-10,000$ & $14 \pm 6$ & $11 \pm 7$ & $10 \pm 7$ & $18 \pm 10$ \\
$10,000-20,000$ & $4 \pm 3$ & $5 \pm 4$ & $4 \pm 3$ & $5 \pm 4$ \\
$20,000-50,000$ & $11 \pm 5$ & $11 \pm 5$ & $11 \pm 5$ & $10 \pm 5$ \\
$>50,000$ & $31 \pm 6$ & $32 \pm 6$ & $34 \pm 6$ & $35 \pm 6$ \\
\hline
\end{tabular}




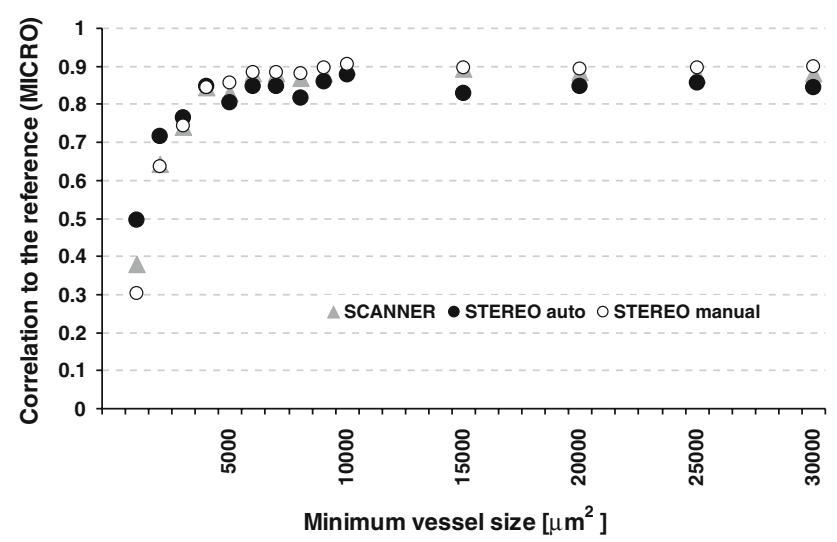

Fig. 2 Variation of the correlation between MVA time series of the reference (MICRO) and the other procedures in dependence on minimum vessel size. Values correspond to survey performed on two cores of the same tree $(n=102)$

any other procedure, since smaller vessels were detected. In fact, all procedures yielded similar numbers of vessels if only those larger than $6,000 \mu \mathrm{m}^{2}$ were considered.

The correlations between the MVA time series of the fast procedures and the reference (MICRO) considerably increased when raising the vessel size threshold. For vessels $>6,000 \mu \mathrm{m}^{2}$ the correlation coefficient stabilized at values of $0.85-0.90$ (Fig. 2). This indicates that any further increase in the minimum vessel area does not improve the similarity to the reference. Above this size, even an additional correction of misrecognized vessels (STEREOmanual vs. STEREO-auto) only marginally $(r \leq 0.02)$ improved the correlation with the reference.

\section{Measurement error}

The ME of the fast procedures depended on the vessel size threshold applied (Fig. 3). Differences in MVA values

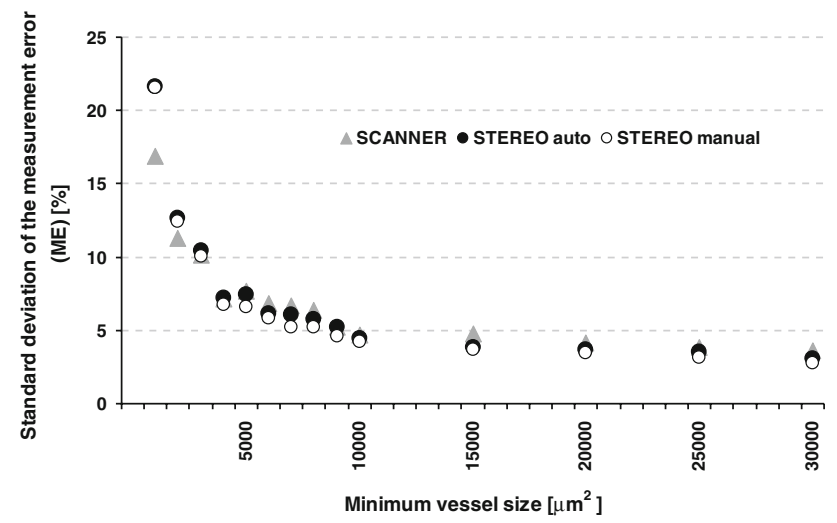

Fig. 3 Variation of the measurement error (ME) by increasing the minimum vessel size. The standard deviation of ME is expressed as the percentage of MVA values of the reference (MICRO) and is based on the survey performed on two cores of the same tree $(n=102)$
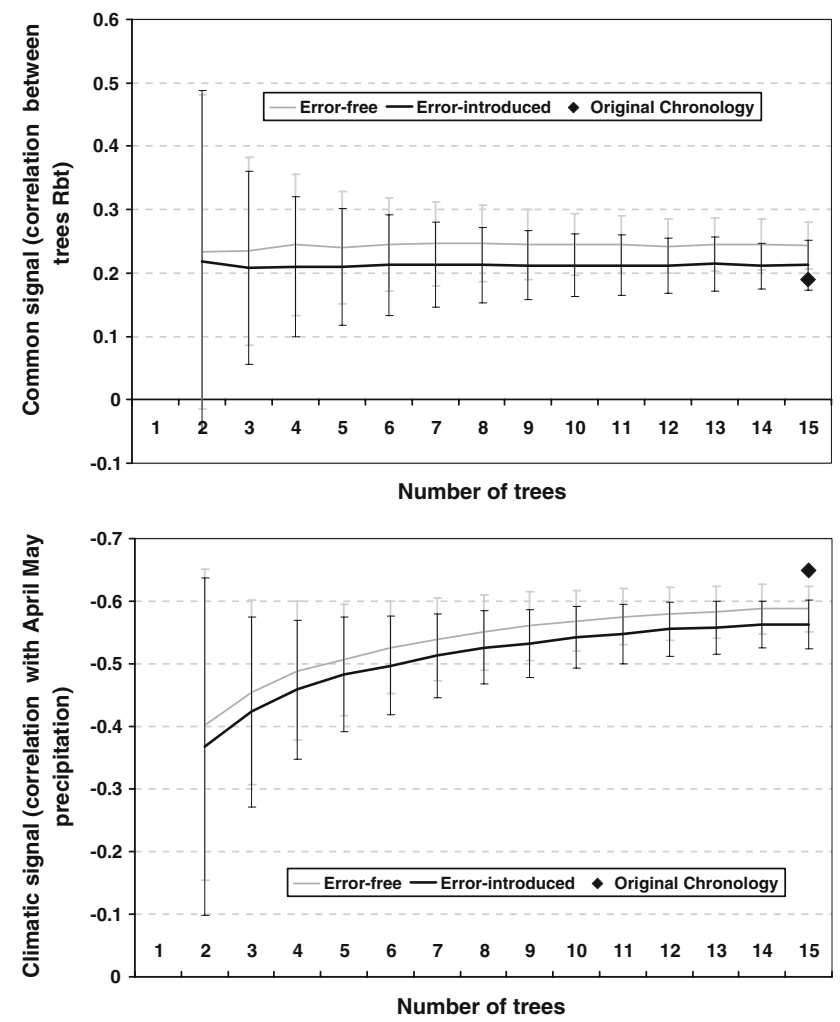

Fig. 4 Variation (mean \pm 1 standard deviation) of the common signal (Rbt) and the climatic signal (correlation with April-May precipitation) for SCANNER while increasing the number of trees considered in the chronology. Values of the error-free and errorintroduced chronologies are calculated with bootstrap procedures ( $n=1,000$ with tree replacement). The introduced error (mean $=0 \%$, standard deviation $=4.669 \%$ of MVA) corresponds to the measurement error calculated for differences between the SCANNER and the reference (MICRO) with the minimum vessel size of $10,000 \mu \mathrm{m}^{2}$. The error was randomly generated for each single MVA value after each repetition. The original chronology corresponds to the chronology obtained with all the 15 trees, without bootstrapping

were larger when the threshold was low, mainly due to the deficient recognition of smaller vessels. The standard deviation of ME expressed as a percentage of MVA of both of the faster procedures stabilized below $\pm 5 \%$ when vessels $>10,000 \mu \mathrm{m}^{2}$ were excluded. Above this size threshold, the MEs did not strongly differ among procedures $(\leq 1 \%)$. SCANNER showed the largest and STEREO-manual the smallest departures from the reference.

Loss of signal information

The original SCANNER MVA chronology (based on the survey for all the 15 trees) showed a mean sensitivity of 0.11 , a common signal of 0.19 , and a highly significant correlation $(-0.65 ; P<0.001 ; n=51)$ with April-May precipitation.

The progressive increase in the number of trees included did not change the mean common signal but did strengthen 
the mean climatic signal, which just seemed to stabilize at a sample size of about 12 trees (Fig. 4). These outcomes slightly differed from what resulted from the original SCANNER MVA chronology, since by bootstrapping we allowed replacement of sampled trees. The introduction of the ME hardly weakened both signal strength components. The loss of common signal due to ME was about $r=0.03$ and the weakening in climatic signal varied from $r=0.01$ to 0.05 depending on how many trees were considered for the calculations.

\section{Discussion}

Consequence of improper measurement quality

The quality of ecologically relevant information extracted from tree-ring series mainly depends on the strength of (1) mean sensitivity, (2) common signal, and (3) environmental signal (Fritts 2001). In other terms, the more sensitively and univocally tree growth responds to the environmental conditions, the higher is the confidence into the tree-ring proxy reconstruction. Inaccuracy of the survey can therefore have different consequences depending on the confidence of the extracted signal (proxy). A small measurement error can have little effect on the reconstruction if the signal is strong, but can mask an existent relationship if the signal is weak.

Generally, time series of anatomical features of water conducting cells show a lower sensitivity and common signal than other tree-ring parameters (e.g., ring width or maximum wood density). Previous surveys on the size of earlywood vessels of ring-porous species showed mean sensitivity and common signal values of sites chronologies ranging from 0.05 to 0.20 and from 0.10 to 0.77 , respectively (García-González and Eckstein 2003; Fonti and García-González 2004; Tardif and Conciatori 2006: Fonti et al. 2007; García-González and Fonti 2008). Nevertheless, in some cases correlations with climatic parameters reached values $>0.60$, especially when only the more responsive earlywood vessels (e.g., the first row of earlywood vessels, García-González and Fonti 2006, 2008) were considered. Still, in cases where the expressed proxy signal is not very strong the quality of the measurement might influence the output.

\section{Causes for inaccurate measurements}

The wood surface preparation applied was insufficient to allow the recognition of vessels $<6,000 \mu \mathrm{m}^{2}$, irrespective of the pixel resolution of the images. Above this threshold, MVA series of all evaluated procedures were very similar to the reference, but a residual variance of $19-28 \%$ between the surface procedures and the reference still remains unexplained.

Part of the unexplained variance may be related to a 1$2 \mathrm{~mm}$ vertical shift of the cross-section plane along the stem axis that unavoidably accompanied the preparation of thin sections. The MICRO images therefore do not exactly correspond to the surface of the other procedures. Since vessels are not perfectly cylindrical and also slightly change their position (Kitin et al. 2004), slight modifications of the vessel conducting area have to be expected (cf. Fig. 4). Other possible reasons for divergences are related to different pixel resolution (pixels are discrete entities; for small objects such as vessels, measuring a given object will produce slightly different area values if the object is represented in different pixel resolutions), or to differences in image processing operations (contrast enhancement, image segmentation, degree of vessel outline smoothing). Finally, differences between STEREO and SCANNER can be explained by the fact that in STEREO the inaccuracy is reduced by excluding marginally truncated vessels by an appropriate delimitation of the area of interest.

Manually correcting misrecognized vessels reduced the unexplained variance by $9 \%$ or less. Omitting manual corrections thus has only a small influence on the result. However, an optimal surface preparation is crucial if no manual correction is going to be performed.

\section{Trade-off between time and accuracy}

Building a chronology of wood anatomical features by means of thin sections is very time-consuming. For each year of the chronology as many as possible representative cells from rings of different trees have to be measured.

The present work performed on vessels that contain a strong precipitation signal demonstrates that measurements of ring-porous earlywood vessels performed on images captured directly from the wood surfaces reduce the measurement time by more than 20 times as well as the requirements for infrastructure (e.g., sliding microtome), essentially without loosing any signal information. Although the ME contains a systematic component due to an unavoidable vertical shift in the plane of the crosssections of the reference, and although we chose a very conservative approach by deliberately performing the analysis with the least accurate procedure (SCANNER) which excluded manual correction of misidentified vessels, the loss in common and climatic signal was very small. This finding is especially remarkable if we also consider that a modest increase in sample size can strengthen the signal and thus fully compensate for the inaccuracy of the survey. These new surveys are still considerably more time-efficient than MICRO, although an additional number of trees needs to be measured. 
The advantage of these simplified surveying procedures, however, is currently limited to vessels $>6,000 \mu \mathrm{m}^{2}$. For the earlywood vessels of ring-porous wood species like sessile oak ( $Q$. petraea), this size threshold does not represent an obstacle since it corresponds to the small earlywood vessels at the transition to the latewood. Moreover, as it appears from the previous studies on ringporous earlywood vessels, the climatic signal mainly resides in the larger earlywood vessels (García-González and Fonti 2006), mostly those $>10,000 \mu \mathrm{m}^{2}$ (Fonti and García-González 2004; Fonti et al. 2007) or $5,000 \mu \mathrm{m}^{2}$ (García-González and Eckstein 2003). When including smaller vessels (e.g., $>1,200 \mu \mathrm{m}^{2}$, cf. Tardif and Conciatori 2007) the environmental signals might get masked, since the larger number of vessels considered attenuates the relative weight of the larger and more responsive ones (e.g., Sass and Eckstein 1995; García-González and Eckstein 2003).

However, improvements in wood preparation techniques will likely contribute to lower the current minimum vessel size, rendering the survey possible for vessels of both latewood of ring-porous and diffuse-porous tree species.

With regard to our results, we conclude that a measuring procedure that combines advanced wood preparation and image capturing techniques with automated image analysis can achieve a very efficient measurement of earlywood vessels of ring-porous species, without loosing significant signal information. Altogether, our study may significantly promote the applications of quantitative wood cell anatomy in ecological studies.

Acknowledgment This project was supported by the Swiss National Science Foundation (project INTEGRAL) and by the European Union (project MILLENIUM).

\section{References}

Briffa KR, Jones PD (1992) Basic chronology statistics and assessment. In: Cook ER, Kairiukstis LA (eds) Methods of dendrochronology: applications in the environmental science. Kluwer, Boston, pp 137-152

Cook E, Briffa K, Shiyatov S, Mazepa V (1992) Tree-ring standardization and growth trend estimation. In: Cook ER, Kairiukstis LA (eds) Methods of dendrochronology: applications in the environmental science. Kluwer, Boston, pp 104-123

Evans R, Downes G, Menz D, Stringer S (1995) Rapid measurement of variation in tracheid transverse dimensions in a radiata pine tree. Appita J 48:134-138
Fonti P, García-González I (2004) Suitability of chestnut earlywood vessel chronologies for ecological studies. New Phytol 163:7786. doi:10.1111/j.1469-8137.2004.01089.x

Fonti P, Solomonoff N, García-González I (2007) Earlywood vessels of Castanea sativa record temperature before their formation. New Phytol 173:562-570. doi:10.1111/j.1469-8137.2006.01945.x

Fritts HC (2001) Tree rings and climate. Academic Press, New York

García-González I, Eckstein D (2003) Climatic signal of earlywood vessels of oak on a maritime site. Tree Physiol 23:497-504

García-González I, Fonti P (2006) Selecting earlywood vessels to maximize their environmental signal. Tree Physiol 26:1289-1296

García-González I, Fonti P (2008) Ensuring a representative sample of earlywood vessels for dendroecological studies: an example from two ring-porous species. Trees (Berl) 22:237-244. doi: 10.1007/s00468-007-0180-9

Holmes RL (1983) Computer-assisted quality control in tree-ring dating and measurement. Tree Ring Bull 43:69-78

Jagel R, Telewski FW (1990) Image analysis. In: Cook ER, Kairiukstis LA (eds) Methods of dendrochronology: applications in the environmental science. Kluwer, Boston, pp 76-93

Kitin PB, Fujii T, Abe H, Funada R (2004) Anatomy of the vessel network within and between rings of Fraxinus lanuginosa (Oleaceae). Am J Bot 91:779-788. doi:10.3732/ajb.91.6.779

Munro MAR, Brown PM, Hughes MK, Garcia EMR (1996) Image analysis of tracheid dimensions for dendrochronological use. In: Dean JS, Meko DM, Swetnam TW (eds) Tree rings, environment, and humanity: proceedings of the international conference, Tucson, Arizona 17-21 May 1994. Radiocarbon, The University of Arizona, Tucson, pp 843-851

Panyushkina P, Hughes M, Vaganov E, Munro M (2003) Summer temperature in northeastern Siberia since 1642 reconstructed from tracheid dimensions and cell numbers of Larix cajanderi. Can J Res 33:1905-1914. doi:10.1139/x03-109

Park WK, Telewski FW (1993) Measuring maximum latewood density by image-analysis at the cellular-level. Wood Fiber Sci 25:326-332

Pumijumnong N, Park WK (1999) Vessel chronologies from teak in northern Thailand and their climatic signal. IAWA J 20:285-294

Sass U, Eckstein D (1995) Preparation of large thin-section and surface of wood for automatic image-analysis. Holzforschung 48:117-118

Schweingruber FH (2001) Dendroökologische Holzanatomie. Paul Haupt, Bern

Spiecker H, Schinker MG, Hansen J, Park YI, Ebding T, Doll W (2000) Cell structure in tree rings: novel methods for preparation and image analysis of large cross sections. IAWA J 21:361-373

St George S, Nielsen E, Conciatori F, Tardif JC (2002) Trends in Quercus macrocarpa vessel areas and their implications for treering paleoflood studies. Tree Ring Res 58:3-10

Tardif JC, Conciatori F (2006) Influence of climate on tree ring and vessel features in red oak and white oak growing near their northern distribution limit, southwestern Quebec, Canada. Can J Res 36:2317-2330. doi:10.1139/X06-133

von Arx G, Dietz H (2005) Automated image analysis of annual rings in the roots of perennial forbs. Int J Plant Sci 166:723-732. doi: $10.1086 / 431230$ 